\title{
A study of deformation and phase transformation coupling for TRIP-assisted steels
}

\author{
Ma, Anxin, anxin.ma@rub.de; Koester, Aenne; Gupta, Satya;
} Hartmaier, Alexander, Ruhr-University Bochum, Germany

\begin{abstract}
A constitutive model for transformation induced plasticity (TRIP)-assisted steels is proposed that considers the elastic-plastic deformation of ferrite and austenite, the austenite-martensite phase transformation and the elastic deformation of martensite. Within this model, an explicit relation between martensite nucleation and plastic deformation within an austenite grain has been established based on the inverse Nishiyama-Wassermann relationship. In particular, strain-induced martensite nucleation and stress-assisted martensite growth have been included in one model with the help of a thermodynamic principle. Furthermore, the formulation of the stress-assisted martensite growth has been enhanced to include commonly observed physical mechanisms influencing the martensite transformation and global mechanical behavior of low alloyed TRIP-assisted steels. With this model, we found consistently with experiment that the TRIP effect enhances the effective work hardening rate and hence is beneficial for improving strength and ductility of steels. The mechanical anisotropy produced by stress-assisted and strain-induced phase transformations are significantly different. We observed that austenite grains transform to martensite more quickly under tension than under compression. Furthermore, the influence of alloy composition, martensite lamellar structure, transformation rate and active transformation systems on the strength and ductility of low alloyed TRIP-assisted steels has been investigated. We find that this refined model reliably captures the influence of alloy composition, temperature, and martensite lamellar structure. It is shown that the choice of the orientation relationship between austenite and the martensite does not significantly influence the global mechanical behavior of TRIP-assisted steel.
\end{abstract}

